\title{
Entretien avec Pascale Kramer
}

\author{
Ursula BÄHLER \\ Zurich \\ Jean-Philippe Coen \\ Zurich \\ Pascale KrAmER \\ Paris
}

\begin{abstract}
Artiste invitée de la Chaire de littérature et culture française de l'École polytechnique fédérale de Zurich, Pascale Kramer a donné à l'automne 2019 un cours intitulé "Cheminement autodidacte en écriture ». Celui-ci aura été l'occasion de revenir sur les textes, les films et les expériences de vie qui ont nourri son travail de romancière. C'est dans ce cadre qu'elle a souhaité partager avec son amie, l'auteure Ghislaine Dunant, une soirée consacrée à l'écriture de la sexualité. L'entretien qu'on lira ici y fait allusion. Réalisé le 26 novembre 2019 dans les bureaux de l'École polytechnique, cet entretien a été revu par Pascale Kramer. Si elle a procédé aux coupes jugées indispensables pour supprimer certaines répétitions et alléger l'ensemble, sa relecture a principalement visé à prolonger la réflexion initiale, à en préciser les lignes et les enjeux. L'ensemble garde cependant quelque chose de sa parole, de son rythme et de sa force. Que Pascale Kramer soit chaleureusement remerciée d'avoir accepté de poursuivre un dialogue amical initié il y a quelques années déjà lors de rencontres au Romanisches Seminar de l'Université de Zurich. Un grand merci à Sophie Hochuli qui a assuré la transcription.
\end{abstract}

Keywords : Pascale Kramer, roman contemporain, littérature francophone, écriture et technique romanesques, éthique, réception critique, monde de l'édition

VERSANTS (V, Ursula Bähler et Jean-Philippe Coen) : Pour commencer voudrais-tu nous dire comment tu es venue à l'écriture, ce qui t'a poussée au départ et ce qui te pousse encore aujourd'hui à t'investir dans l'écriture?

PASCALE KRAMER (PK) : C'est une question difficile parce que je ne me souviens pas d'un temps où je n'écrivais pas. Déjà toute gamine, j'écrivais, j'inventais des histoires, j'avais plein d'idées. Puis à l'adolescence, j'ai fait des textes plus personnels, des trucs d'ados. Je ne me souviens plus très bien quelle était l'impulsion des tout premiers, ceux qui sont parus aux Éditions de l'Aire, elle était en tout cas très différente de celle des livres écrits ces dernières années. En relisant Manu pour préparer ce cours à l'École polytechnique de Zurich, je me suis dit que ce n'était plus la même âme qui l'avait écrit. Mes livres suivent mon évolution personnelle, forcément. Je sais 
très bien ce qui me pousse à écrire aujourd'hui. L'écriture me permet de mieux comprendre le monde, je crois que c'est vraiment ça...

\section{$V$ : Parce que l'écriture serait une façon d'y mettre un certain ordre...}

PK : Non, je n'essaie pas de réparer quoi que ce soit. Mais derrière chaque roman, il y a une interrogation humaine. En tout cas, pour les dix derniers. Écrire me permet de vivre d'autres vies, de me mettre dans la peau d'autres que moi, de comprendre ce qui leur arrive. Le meilleur exemple en est L'Adieu au Nord, mon plus grand insuccès d'ailleurs ! Vivant alors dans un bled paumé du Nord de la France où il y avait beaucoup de précarité et de violence, j'avais eu une vraie intuition de ce qui pousse des hommes pas suffisamment respectés dans leur vie, pleins de frustration vis-à-vis des femmes, à en venir à frapper leur compagne. Je me suis mise à la place de l'un d'eux pour tenter de comprendre et de... - non pas excuser - mais faire comprendre. Je le disais au public du cours, je rentre dans la peau de mes personnages. C'est plus une démarche de comédien qui m'anime.

\section{$V$ : D’ò̀ viennent les sujets de tes romans? Ça te tombe dessus ou...}

PK : Oui, tout à coup, il y a une interrogation ou une intuition qui me retient. Par exemple pour Autopsie d'un père, c'était la montée de ce qu'on appelle les nouveaux réacs. Puis il y a eu l'affaire Richard Millet et son Éloge littéraire d'Anders Breivik, jeune néonazi qui a commis un massacre en Norvège. Millet a été complètement mis au ban de la société. Nous avons tous signé une lettre ouverte parue dans Le Monde, initiée par Annie Ernaux. Je n'en ai pas été très fière, nous ne faisions que tirer sur l'ambulance. Et puis j'avais connu Richard Millet, un homme instruit et intelligent, et je me demandais ce qui l'avait amené à écrire des choses comme ça. J'ai eu envie d'appréhender ça aussi à travers un roman. C'est ainsi à chaque livre, j'ai l'intuition de quelque chose et je la creuse.

$V: A u$ fil des livres ces intuitions changent. Il y a une certaine évolution de livres en livres. Comment tu décrirais ce parcours?

PK : Mes sujets ont évolué avec mes interrogations de citoyenne. Je suis devenue beaucoup plus politisée. En allant vivre dans le Nord de la France, j'ai découvert le monde de la précarité de plus près. Et le monde lui-même a pas mal changé. J'ai été très peu politisée quand j'étais plus jeune, mais je pense que les enjeux, en tout cas en Europe, n'étaient pas du tout les mêmes qu'aujourd'hui. Alors oui, peut-être que ma conscience a évolué parce que les enjeux sont plus importants maintenant que dans ma jeunesse. Je n'es- 
saie pas pour autant de délivrer des messages, encore moins de faire changer les opinions, mais de comprendre de plus près ce qui se passe.

$V:$ Est-ce que tu lis ce qu'on publie sur tes romans?

PK : Oui, oui, je lis...

\section{$V$ : Quel est ton rapport à cette forme de critique journalistique?}

PK : J'ai la chance de n'avoir encore jamais été esquintée, pour la simple raison que je ne suis pas suffisamment connue pour que les éreinteurs s'acharnent sur moi ! et tant mieux... Après, je ne me sens pas toujours forcément entendue, surtout pour mes livres les plus politiques. J'ai l'impression qu'ils ont été pas mal... - non pas caricaturés -, mais qu'on en a fait une lecture critique orientée là où j'essayais de mettre de la nuance.

\section{$V$ : Une lecture un peu simplifiée?}

PK : Oui, voilà, un peu simplifiée.

\section{$V:$ Dans quel sens?}

PK : Par exemple, Autopsie d'un père. Le personnage principal est un journaliste conspué pour avoir défendu des jeunes qui ont commis un meurtre sur un migrant. Mais je n'en ai pas fait un monstre pour autant, cela n'aurait eu aucun intérêt romanesque. Ce livre, pour lequel je n'ai eu que des critiques en Suisse, a été interprété comme une prise de position contre ces dérives fascisantes, alors que j'avais cherché à comprendre et donc à mettre de la nuance. Je ne formule pas de jugements de valeur. Même des choses évidemment choquantes comme la violence à l'égard des femmes ou des migrants m'interroge et j'essaie de faire partager cette interrogation dans mes romans en allant puiser dans toute la gamme des émotions qu'on a tous en soi. Or j'ai l'impression que les gens y plaquent leur propre opinion. Parce que je ne donne pas beaucoup de clés non plus, c'est sûr !

$V:$ Pour tes autres romans, il y a eu des recensions dans la presse française. Autopsie d'un père, c'est une exception.

PK : J'ai eu deux gros insuccès notoires. L'Adieu au Nord, même s'il y a eu un très beau texte de Christophe Ono-dit-Biot dans Elle. Et Autopsie d'un père, pour lequel il n'y a rien eu du tout côté français. C'est très difficile à vivre pour un auteur. Nous avons besoin de critiques, c'est un besoin de recon- 
naissance. Après cet insuccès pour Autopsie d'un père, j'ai reçu le Grand Prix suisse de littérature 2017 , ça m'a fait un bien fou... Tout d'un coup, je me suis dit : «Mais oui quand même j'ai écrit plus d'une dizaine de livres... Il y a une cohérence et ce travail est validé ». Il faudrait être beaucoup plus philosophe face aux insuccès, mais on a l'impression de disparaître. Pour Autopsie d'un père, les circonstances ont joué contre moi. J'ai fait le service de presse le lendemain de l'attentat du Bataclan. Tout le monde était en vrac. Les journalistes n'avaient pas forcément envie de se plonger dans une histoire de nouveaux réacs, alors que la période était si sombre.

\section{$V$ : Est-ce que pour L'Adieu au Nord tu t'expliques le silence de la critique?}

PK : Le livre n'a tout simplement pas été ouvert, ou seulement entrouvert. C'est ce que j'ai fini par apprendre de mon éditrice de l'époque. J'avais des difficultés avec le Mercure de France. Je n'avais jamais de retour sur la réception du livre, sauf quand sortaient des articles positifs. Le livre n'avait aucune presse, mais tout le monde faisait comme si de rien n'était. Un jour, j'ai posé la question à Isabelle Gallimard qui, se sentant acculée, m'a fait une réponse assez violente : «Il faut quand même être conscient de ce qu'on écrit ». Et de fait, cette histoire de cressonnière du Nord, ça n'intéressait pas les journalistes. En tant qu'auteur, on interprète toujours le silence de la presse comme : «Les journalistes n'ont pas aimé le livre ». Non. Le plus souvent c'est qu'ils ne l'ont pas lu. Il faudrait vraiment rester lucide sur tout ça, mais on a besoin de recensions. J'ai un ami, l'écrivain Anouar Benmalek, qui me disait un jour en riant : "Je peux faire le deuil d'un livre une fois que j'ai eu un article dans Le Monde ». Heureusement, on finit par se ressaisir. J'ai compris pourquoi certains de mes romans ont plus de succès que d'autres ce qui me donne à penser que je ne serai jamais un bestseller - : ce sont ceux où les lecteurs peuvent s'identifier à un personnage, manifester de l'empathie pour celui-ci, comme dans Les Vivants, par exemple.

\section{$V: \grave{A}$ l'instar aussi d’Une famille, plus récemment?}

PK : Oui, typiquement. Beaucoup de gens se sont reconnus dans cette histoire et se sont sentis proches des personnages. Les gens sont mal à l'aise, souvent, avec mes livres. Je m'en aperçois quand je vais dans des rencontres en médiathèques où la plupart des personnes m'ont lue parce que je suis annoncée au programme, mais pas forcément parce qu'elles ont été attirées par mon livre. On vient me dire : « Mais c'est noir, ça me met mal à l'aise. » Pourtant il y a plein de livres beaucoup plus noirs que les miens. Je vois très bien ce qui met le lecteur mal à l'aise, et c'est précisément là où j'ai envie d'aller. 


\section{$V$ : Faire mal au lecteur?}

PK : Non! mais j'ai besoin d'aller voir là où ça fait un peu... un peu mal. Comme lectrice, j'aime les livres qui m'égratignent, qui me bousculent, qui vont me chercher dans mes zones d'ombre.

\section{$V:$ Tu nécris pas pour le lecteur?}

PK : Non. Franchement non. Quand je suis dans le livre, j'essaie seulement de creuser mon intuition, d'avancer, de me dire que, là, je suis juste. C'est uniquement une fois le livre terminé, que je me pose la question : «Quelle réception, ça va avoir?»

\section{$V$ : Est-ce que tu peux nous dire un peu comment prend forme un livre?}

PK : Ça démarre par un sujet. Je vais vous donner l'exemple du nouveau réac. J'ai ce personnage que j'identifie à peu près, physiquement, sa personnalité. Ensuite je cherche le personnage à travers les yeux de qui je vais le regarder. Car je suis toujours dans une subjectivité. Je n'écris jamais au-dessus de mes personnages. Donc, je ne voulais pas être dans sa perspective à lui - sauf dans le premier chapitre qui permet de nous faire appréhender ce qu'il vit de l'intérieur. C'est d'ailleurs un chapitre qui m'a donné beaucoup de mal. J'ai décidé de le montrer à travers les yeux de sa fille, une fille qui a souffert de son ironie, qui s'est toujours sentie insuffisante vis-à-vis de lui. Puis me vient la première scène : lui dans le train qui aperçoit sa fille en train de pleurer en silence à l'autre bout du wagon, et son petit-fils qui la prend soudain dans ses bras. C'est d'ailleurs une scène à laquelle j'ai assisté dans un car Air France avec un ami. J'avais envie d'utiliser cette scène bouleversante, du fait de l'incroyable gravité du petit garçon soucieux de sa mère. J'avais donc cette première scène, et les lieux. La maison, c'est la maison d'amis en Normandie. Ensuite il y a les scènes les plus marquantes et les autres personnages qui prennent forme. Et il y a des surprises. Par exemple, je ne sais plus pourquoi j'ai voulu que ce petit garçon soit sourd. C'était sur le plan romanesque une bonne idée, cela a permis de faire ressortir certains traits de caractères des autres personnages.

\section{$V: T u$ prends des notes?}

PK : Non, jamais. 
$V$ : Donc, ça commence avec la première phrase de la première scène...

PK : Non, pas la première phrase, la première scène. Et après les personnages, et une couleur aussi, chaque livre a une couleur dominante.

$V$ : Tout ça c'est dans ta tête?

PK : C'est dans ma tête, oui, et ensuite seulement je commence à écrire. Manu est le premier roman que j'ai écrit d'une seule traite, le premier qui ait été publié après cinq manuscrits qui n'ont jamais trouvé d'éditeur. À l'époque, cela me paraissait injuste. Mais ces textes, pour lesquels j'avais vaguement un thème de départ, après quoi je laissais courir mon imagination, n'étaient pas suffisamment tendus, il n'y avait pas de dramaturgie. Même s'il n'y a pas de grande dramaturgie aujourd'hui dans mes livres, il y a une tension. Manu, je l'avais écrit d'une traite, puis je l'ai énormément retravaillé. Je me suis dit que ce n'était pas mal de poser l'histoire d'un coup et de revenir dessus. J'ai répété le procédé pour Retour d'Uruguay et plusieurs autres livres. À partir de L'Implacable Brutalité du réveil, je me suis sentie plus libre, et j'ai écrit chapitre par chapitre, mais en sachant où j'allais. J'ai acquis suffisamment de maîtrise pour arriver à faire un livre qui reste tendu, même sans l'écrire d'une seule traite.

$V:$ Quand tu corriges, tu effaces, tu réécris des phrases entières, ou ce sont des détails, ou...?

PK : Je cherche la justesse.

\section{$V:$ De chaque mot?}

PK : Oui, de chaque mot pour qu'il traduise avec justesse l'image que j'ai en tête. J'ai participé l'autre jour à une rencontre de la Fureur de lire autour de la dyslexie (je suis une immense dyslexique). Dans la salle, il y avait des logopédistes qui m'ont appris que les dyslexiques voient en images et non pas en mots. Ça m’a éclairée sur ma façon de travailler et sur le fait que je ne prends pas de notes. Ce sont des images que j'ai en tête, pas des mots. Je fais confiance aux images qui me restent quand je construis mon livre mentalement. J'en discute avec Marie Nimier chez qui je vais travailler souvent. Elle fonctionne très différemment, elle s'appuie vraiment sur les mots, leur musique, leurs multiples sens. Elle prend énormément de notes et elle fait confiance à la phrase. Moi j'essaie de restituer des images, des sensations, 
des sentiments tels que dans la vie. Ensuite je travaille, travaille jusqu'à ce que les mots fassent surgir ces images.

\section{$V$ : C’est extrêmement précis, quand même!}

PK : Oui. Mais jamais je ne choisirais un mot pour sa sonorité, ou pour ce qu'il peut se comprendre dans plusieurs sens, je le choisis uniquement parce qu'il exprime exactement. J'essaie de me mettre à la place du personnage et de voir ce qui est juste par rapport à ce qu'il pense ou ressent. Est-ce qu'il y a de l'ambivalence ? Est-ce qu'il y a de l'ambiguïté ? C'est vrai aussi que je travaille beaucoup le rythme de la phrase et que je condense beaucoup. Comme je dis souvent : quand tu rentres dans une pièce, tu as instantanément une foule d'impressions, selon qu'il y a du monde ou non, si l'ambiance est hostile ou amicale. Tout ça se passe en une fraction de seconde, alors qu'il faudrait plusieurs phrases pour le décrire. Pour rendre cette instantanéité, je fais des raccourcis, des juxtapositions inhabituelles de mots qui produisent un effet. C'est une leçon que j'ai tirée de Quignard.

$V$ : Pendant l'écriture du livre ou, plus tard, au moment de la correction $d u$ premier jet, est-ce que tu procèdes à des déplacements de chapitres, de parties?

PK : Non, presque pas.

\section{$V$ : Pour Manu, par exemple, le début et la fin qui se répondent?}

PK : C'était comme ça dès le départ. Non, je ne fais pas de grands changements de structure, je coupe peu. En revanche, je rajoute de la matière, de la profondeur. Si j'écris d'un seul jet toute une scène ou un chapitre entier en laissant courir les doigts sur le clavier, il m'arrive de retirer des paragraphes inutiles ou creux. Sinon je rentabilise relativement tout ce qui vient, en remettant du sens et de la matière là où c'est trop facile.

$V$ : Tes livres sont relativement courts. Es-tu tentée par un format plus long, une épopée de cinq cents ou six cents pages?

PK : En ce moment, je travaille à un livre plus long ... En tout cas, je me prends plus de temps. Mais j'ai besoin de pouvoir visualiser le livre entier, c'est évidemment plus difficile avec un livre long.

$V: T u$ nous dis que tu gardes tout en tête, alors quand tu te mets à écrire un livre, ce doit être de manière très régulière? 
PK : Non, pas du tout. Je travaille en indépendante, je suis obligée de répondre aux urgences. J'ai toujours travaillé et écrit en parallèle, en tachant de me garder des semaines par ci par là pour écrire. Depuis que Marie Nimier offre chez elle, dans sa grande maison en Normandie, une résidence d'écrivains, je me bloque une semaine tous les un mois et demi, à peu près. Et je réserve ces périodes pour l'écriture, en calant mon travail alimentaire avant de m'y rendre.

\section{$V:$ Quand tu relances l'écriture, tu retrouves les images dans ta tête?}

PK : Oui, et je les redécouvre presque comme neuves. J'ai des amis qui travaillent tous les jours. Je ne pense pas que je le pourrais, je n'aurais plus le recul, je finirais par ne plus voir les choses, d'autant plus que je me relis énormément! Je change un mot dans une phrase, et je relis tout le paragraphe! Je me relis, jusqu'à l'écœurement parfois. C'est pour cela que j'ai besoin de lâcher le livre quelques temps pour le redécouvrir plus tard, avec le plaisir d'y revenir.

\section{$V:$ Tu relis sur l'écran?}

PK : Toujours. Ensuite, de temps en temps, je fais des impressions. Ou je relis sur tablette, pour avoir un autre miroir de page, donc un autre découpage des phrases.

\section{$V:$ Conserves-tu des traces de certains états?}

PK : Non, rien du tout.

\section{$V$ : Rien du tout?}

PK : Non, non, jamais. Je sais qu'il y a des chercheurs qui aiment comparer les différents états d'un même texte.

$V$ : Ils seront déçus!

PK : Bah non!

\section{$V: Y$ a-t-il des moments privilégiés d'écriture dans une journée?}

PK : Non. Il y a en revanche de mauvais moments : cinq heures du soir, mais je pense que c'est pareil pour tout le monde. Même les chiens, m'a-ton dit, ont des crises d'angoisse à cinq heures du soir! Il y a les périodes 
creuses, et celles où je traine, comme le matin. J'ai mon petit rituel, je prends un bon petit déjeuner, je fais de la gym. Ensuite je m'y mets. Le soir, c'est bien aussi. Mais je ne suis pas du genre à travailler toute la nuit.

$V$ : Pour Retour d'Uruguay, tu as évoqué, dans ton cours, la réécriture du livre après avoir essuyé le refus de la directrice de collection de Calmann-Lévy. Y a-t-il eu une forme particulière de réécriture pour ce roman? Comment l'expliques-tu?

PK : C'est mon premier livre un peu politique. C'était l'époque où Le Pen montait dans les sondages et séduisait surtout beaucoup de jeunes hommes. Il m'a semblé avoir une assez bonne intuition de ce qui pouvait être attrayant et attirant chez un homme a priori assez vulgaire, très sexuel en quelque sorte. Raphaël, le personnage central de Retour d'Uruguay, n'est pas un Le Pen, cela aurait été vraiment trop caricatural, mais c'est un homme ambigu et très contestable. Je n'étais pas tout à fait au clair avec ce personnage, ou plutôt avec mon projet de montrer l'attrait qu'il exerçait, et je suis restée dans un entre-deux. Je l'ai fait lire à Martine Saada, mon éditrice à l'époque. Elle a été très mal à l'aise avec ce livre : «Tu as l'air de défendre ce type, on ne comprend pas ce que tu cherches à nous dire ». En fait, le livre n'était pas abouti, parce que je n'étais plus juste par rapport à mon sujet. Martine a refusé le livre, ce qui a été un choc. Puis finalement, je l'ai repris, en tâchant de rester fidèle à mon projet de départ et de l'assumer. Les scènes sont restées les mêmes, mais l'angle avait changé. Tout ça remonte à loin ! Je me souviens aussi d'une remarque de mon ami Jean-Luc Badou, un des initiateurs de la revue Le Passe-muraille et collaborateur des Éditions de L'Aire à l'époque où j'y ai publié mes premiers textes. Jean-Luc Badou a lu absolument tous mes manuscrits, même ceux qui n'ont jamais été publiés. Et il m'avait dit quelque chose de très précieux pour ce personnage de Retour d'Uruguay : "La fascination que Raphaël exerce sur le jeune homme par qui on voit l'histoire est plus dite, formulée, que vraiment vécue. »C'était très juste. Dans le roman publié, il y a des scènes ajoutées suite à cette remarque, comme celle où les deux hommes discutent autour du barbecue puis vont visiter à la lampe de poche une carcasse d'immeubles en construction. Elles rendent bien cette espèce de camaraderie virile, qui valorise le jeune Adrien et qu'il recherche.

\section{$V$ :Au fond, tu t'es plongée plus encore dans l'histoire?}

PK : Oui, en essayant d'être au plus près des personnages que j'avais envie de mettre en scène. Isabelle Gallimard, à qui j'avais donné à lire le texte retravaillé, a tout de suite très bien senti ce que j'avais cherché à faire, et s'est montrée à l'aise avec le livre. C'est comme ça que j'ai changé d'éditeur. 
$V$ : Pour le roman L'Adieu au Nord, tu as parlé dans ton cours de son titre pour lequel il avait été difficile de se mettre d'accord avec l'éditeur. Est-ce que le titre d'un livre arrive très vite au moment de l'écriture ? Avant que celle-ci ne commence ? À la fin?

PK : J'ai toujours un titre dès le début, qui indique quel est le projet, mais qui n'est pas forcément le titre définitif. C'est là où les éditeurs mettent leur patte. Pour Les Vivants, le titre s'est imposé tout de suite. Pourtant c'est l'histoire d'un deuil, mais d'un deuil qui frappe des très jeunes qui n'ont qu'une hâte, c'est oublier le drame. Comme le titre était déjà pris, mon éditeur a obtenu qu'on puisse le réutiliser. Autre exemple : Une famille. Au départ, j'avais un autre titre : La Rémission. Mon projet de départ était de décrire la rechute d'un personnage de très, très grand alcoolique après une période d'abstinence. En cours d'écriture, le projet a passablement évolué. J'ai eu l'idée de changer de perspective à chaque chapitre, donc de personnage par qui l'histoire est vue. C'est en commençant le troisième chapitre que je me suis rendu compte que j'étais en train de faire le portrait d'une famille. Mon éditrice n'était pas totalement emballée par le titre Une famille. C'était trop factuel, trop simple à ses yeux. Finalement j'ai tenu bon. Avec le visuel que l'éditeur a choisi et que je trouve extrêmement fort, cela fonctionne vraiment bien.

$V:$ Pour Le Bateau sec, te rappelles-tu la façon dont ce titre assez étrange s'est imposé?

PK : Oui, c'est une phrase du livre, un livre que j’ai carrément oublié. C'est sans doute la première fois qu'on m'en reparle. Le livre est parti d'un rêve. Je m'étais réveillée avec ce lieu qui pourrait être en Islande, et cet hôtel au milieu de nulle part. Je ne sais plus très bien quel était l'enjeu du livre. Il était très visuel. Tous mes livres sont toujours très visuels d'ailleurs.

$V$ : Encore une question par rapport à la publication. Qu'en est-il de la quatrième de couverture? C'est un exercice difficile aussi, non?

PK : C'est rarement moi qui la rédige. Pour Autopsie d'un père, c'est clairement mon éditrice qui l'a écrite, en orientant volontairement le livre vers sa dimension plus politique.

\section{$V$ : Mais alors ça interprète déjà ce que le roman laisse ouvert !}

PK : Oui. Mais je fais confiance à l'éditeur. C'est l'enveloppe : le titre, la quatrième de couverture, le bandeau avec la photographie. Évidemment, si j'étais tout à fait contre, je serais entendue... Pour Autopsie d'un père, la photo de couverture représentait une femme en train d'enterrer des citrouilles. 
C'était incompréhensible et assez laid. Je ne pense pas que cela ait servi le livre. Mais je n'ai pas pu m'y opposer. On ne trouvait pas de visuel, on me disait qu'il fallait montrer un personnage pour qu'on puisse s'identifier. Finalement l'attachée de presse a dit : «J'aime bien, c'est intrigant » et c'est ce visuel qui est resté !

$V:$ Il y a relativement peu de dialogues dans tes romans. Préfères-tu la mise en scène descriptive?

PK: Oui, c'est vrai. C'est idiot, mais j'aime que les pages soient compactes.

\section{$V:$ Visuellement?}

PK : Oui, il faut que le texte soit compact visuellement.

\section{$V$ : Une manière de ralentir la lecture?}

PK : Non, mais de donner d'emblée une impression de densité. Récemment, j'ai compris que je pouvais intégrer des dialogues sans mettre de guillemets. Comme mes livres sont écrits à l'imparfait, quand on passe au présent, ou qu'il y a tout à coup un je, on comprend qu'on est dans du dialogue direct. Avant, j'utilisais plutôt le dialogue indirect. Directs ou non, les dialogues restent toujours plutôt rares. Je ne pense pas que ce soit par le dialogue qu'on se révèle le plus, parce que c'est ce qu'on contrôle le mieux. C'est plutôt dans les attitudes, dans la façon dont on se tient, dont on s'habille qu'on se définit, bien plus que par les propos.

$V:$ Le décor dans lequel tu plonges les personnages est-il également pour toi un bon révélateur?

PK: Le décor est très important, bien sûr. Il faut que les lecteurs puissent visualiser l'endroit où ils se trouvent plongés, sentir l'ambiance, qu'ils puissent se faire une image des personnages, à travers des petits détails qui les situent sociologiquement. Mais sans doute que chaque lecteur s'en fait une image bien différente de celle que j'ai en tête.

$V$ : C'est aussi, n'est-ce pas, ce à quoi tu es sensible chez les auteurs que tu lis?

PK : Oui, c'est vrai que j'aime les livres denses, ceux qui nous placent dans le concret des vies et du quotidien, qui nous font entrer dans un univers. 


\section{$V:$ Le théâtre ne serait pas une de tes formes privilégiées?}

$\mathrm{PK}:$ Non.

$V:$ C'est un peu le contraire.

PK : J'ai habité dans le Nord de la France et j'y ai perdu mon compagnon. Là-bas, la tradition veut qu'on garde le corps à la maison pendant quelques jours, et que les gens du village viennent lui rendre visite. Ça avait donné lieu à un psychodrame terrible. Je m'étais dit que ça aurait pu faire une pièce de théâtre, mais j'ai renoncé, ce n'est pas mon registre.

$V$ : Serais-tu d'accord pour dire que ce qui t'intéresse, c'est, au fond, ce qu'on ne peut pas forcément verbaliser? C'est-à-dire ce qui est un peu avant la mise en mots?

PK : Oui, tout à fait. Ou disons que j'essaie d'aller au-delà de la convention des sentiments. Quelqu'un meurt, tu es triste. Ça c'est la convention. Dans les faits ce que tu ressens est mille fois plus nuancé et changeant. On peut être très heureux pour quelqu'un et éprouver un peu de jalousie, quasiment en même temps. C'est ça que j'ai envie de faire voir, non pas pour montrer la noirceur des gens. C'est souvent ce qu'on me dit, que je ne montre que le mauvais côté des gens, alors que c'est ce qui me plaît au contraire et me fascine, qu'on soit à triple, à quadruple fond. Je ne porte jamais de jugements de valeur, je suis curieuse de ce que nous sommes.

\section{$V$ : Un intérêt pour l'ambiguité de la nature humaine?}

PK : Oui, pour son ambiguïté. Donc pour sa richesse.

\section{$V:$ Est-ce qu'il reste des domaines tabous?}

PK: Plutôt des pudeurs. Je pense qu'il n'y pas de tabous, qu'on peut à peu près tout dire. En relisant le livre de Ghislaine Dunant L'Impudeur pour la soirée que j'ai organisée avec elle, j'ai constaté que je reste très timide par rapport aux scènes de sexe. Il y en a parfois, mais jamais aussi directes et crues que ce que fait Ghislaine. Et je ne m'intéresse jamais à la sexualité en tant que telle, mais pour ce qu'elle dit du couple. Les scènes de sexe ont toujours une fonction dans mes livres, ce sont aussi des scènes d'action.

$V:$ Dans Manu, il y a quand même des scènes qui...

PK : Qui, oui, sont assez osées. 


\section{$V$ :...qui sont dérangeantes.}

PK : Dérangeantes, parce qu'elles se passent en présence d'un petit garçon de deux ans. Ça peut paraître naïf, mais je n'ai à aucun instant, en écrivant le livre, pensé qu'on pourrait y voir de la pédophilie. Il n'y en a pas, le couple est simplement très libre, c'est une passion torride, le petit est là, il fait chaud, on se promène moitié nu. Ce gosse, ce gamin était craquant, et Manu, le principal personnage féminin du livre avait un rapport à lui extrêmement sensuel, mais pas du tout déplacé.

$V$ : Pour revenir à l'édition d'un texte, quel serait pour toi l'idéal de travail d'une directrice ou d'un directeur de collection?

PK : La directrice que j'ai actuellement, Alix Penent, n'est pas loin de cet idéal. Elle lit très rapidement le manuscrit une fois qu'on le lui envoie. Elle me dit : «Je le lirai tel jour » et elle le fait ! C'est quelqu'un qui comprend le projet, qui vous permet d'aller plus loin, de déverrouiller certaines choses. Pour Gloria, par exemple, mon premier livre publié chez Flammarion, Alix Penent m'avait fait très peu de remarques, mais elle avait mis le doigt sur ce qui manquait, ce que j'avais mis « sous le tapis » : les circonstances concrètes dans lesquelles mon personnage d'éducateur avait été licencié. Il m'a suffi d'ajouter un deuxième chapitre très court, mais qui remettait toute l'histoire en place. Alix est quelqu'un qui, sans vous apporter forcément la solution, arrive à vous amener au bout de votre idée. Nous nous comprenons parfaitement. Je pige tout de suite ce qu'elle veut dire et je vais même souvent au-delà de ce qu'elle cherche à ajuster.

\section{$V$ : Il s'agit d'un rapport professionnel ou c'est... ?}

PK : ...amical. Non, c'est vraiment professionnel ! Avec Isabelle Gallimard, ma précédente directrice au Mercure de France, nous étions devenues amies, mais, professionnellement, même si c'était une très bonne lectrice, ça ne me satisfaisait pas. C'est important que l'éditeur ait de l'ambition pour le livre, qu'il ait envie que ça marche et qu'il fasse tout pour. Je sens cette implication de la part d'Alix Penent. Avec Autopsie d'un père par exemple, qui a eu une réception vraiment inexistante, Alix en a été aussi déçue que moi. Alix est très exigeante. Elle relit plusieurs fois le texte pour ne rien laisser passer. "Il faut qu'on ait fait le maximum », me dit-elle toujours. Ensuite, tout dépend de la façon dont les étoiles sont alignées! Ce n'est plus entre nos mains, mais il faut au moins qu'on n'ait pas de regret, qu'on se dise qu'on est allé jusqu'au bout. Ça me va bien, moi qui suis très perfectionniste. 
$V$ : Tu te considères comme une auteure francophone? suisse?

PK: Oui, comme un auteur suisse, clairement.

$V:$ Ce sentiment, c'est par rapport à quoi?

PK : Par une certaine proximité avec la nature qui est toujours très présente dans mes livres. Et, également, par un rapport au monde, très protestant, ce qui se perçoit dans mes livres.

$V:$ Mais pas par rapport à la langue qui est franco-française?

PK : Non, ça, je ne pense pas.

$V:$ Il n'y a rien de dialectal, il n'y a pas trace de régionalisme, etc.

PK : Non, parce que ce n'est pas le propos. Si je faisais un livre sur la Suisse, évidemment, j'en mettrais. Mais comme souvent mes romans ne sont pas situés géographiquement, ça n’aurait aucun sens.

$V$ : Pour Flammarion, es-tu une auteure suisse? Ou est-ce que l'éditeur ne fait pas la différence avec les auteurs français?

PK : Non, je ne pense pas que Flammarion me sente suisse dans l'écriture. À vrai dire, je n'en sais rien. J'imagine qu'on me voit comme un auteur parmi d'autres.

$V:$ Pour l'héritage protestant que tu indiquais, est-ce que cela passe aussi par des écrivains suisses dont les univers romanesques te seraient proches?

PK : Davantage par les auteurs américains, chez qui on perçoit aussi l'influence protestante, et dont je me sens plus proche. Quand j'ai découvert la littérature américaine, je me suis dit : «Voilà, c'est ce que j'ai envie de faire ». C'est une façon d'être dans le réel, de s'ancrer dans le quotidien des gens.

$V$ : Est-ce quil y a une solidarité parmi les auteurs suisses? Est-ce qu'on cherche à simposer quand on est auteur suisse? Est-ce qu'on a le désir ou est-ce qu'on voudrait être accepté surtout à Paris ?

PK : Je suis d'une génération qui avait ce complexe parisien, clairement. Je ne le nie pas. Je voulais habiter à Paris. Je voulais être de ce monde que je 
voyais à travers les magazines et la télévision. Tous les francophones, c'est terrible, on a ce besoin d'être reconnus par Paris. La nouvelle génération est beaucoup plus affranchie. Je l'admire. Il y a les éditions Zoé qui ont vraiment réussi à s'imposer en tant qu'éditeur tout court et non pas en tant qu'éditeur suisse. J'étais récemment dans un salon du livre à Montpellier où Alice Zeniter avait une carte blanche et avait invité de jeunes auteurs qui, sans être forcément les plus connus, sont repérés comme des talents nouveaux. Dans une table ronde, l'un d'entre eux a évoqué un livre publié chez Zoé, écrit à plusieurs mains - comme un feuilleton télévisé. Quand je l'ai fait remarquer à une jeune éditrice de chez Flammarion qui était présente, elle m’a répondu : «Zoé fait partie des éditeurs chez qui on a envie de travailler aujourd'hui ». Zoé a vraiment réussi à s'imposer et à imposer ses auteurs.

\section{$V$ : Est-ce qu'il y a une rivalité entre les deux mondes?}

PK : Non, je ne pense pas, mais comme je le disais, la nouvelle génération assume sa suissitude. Elle a aussi imaginé des expériences d'écriture à plusieurs, qui ne me tentent pas du tout cela dit, mais qui apporte quelque chose de nouveau.

\section{$V$ : Stand-by d'Aude Seigne, Daniel Vuataz et Bruno Pellegrino, par exemple?}

PK : Voilà. C'est intéressant ce qu'ils font et on sent une vraie complicité entre eux. Mais ce n'est pas du tout pour moi. Je suis trop lente, pas assez sûre de moi.

$V$ : Que penses-tu de ces instituts, à l'instar de l'Institut littéraire suisse de Bienne?

PK : J'ai suivi en auditrice libre des cours d'écriture de scénarios à Los Angeles. On y enseignait comment écrire des scénarios qui fonctionnent. Je pense que des écoles comme celle de Bienne, donnent des méthodes, des clés, pour réussir un livre qui fonctionne. Ça formate. Je suis plutôt de la vieille école. Il faut que chacun trouve sa propre voix, même si, comme dans mon cas, cela prend des années.

$V$ : Tu parlais tout à l'heure de ton sentiment d'être une auteure suisse, mais, en même temps, tu avais besoin de Paris !

PK : Oui, je tenais coûte que coûte à publier à Paris. Pour moi, il n'y avait pas de salut en dehors de Paris. Alors que je suis tellement Suisse. Étrangement, c'est ce que j'ai compris quand j'ai commencé à faire de longs 
séjours aux États-Unis. Je m'y suis sentie beaucoup plus en terrain connu qu'en France. J'y ai retrouvé ce même sens civique qui vient du protestantisme. Je me suis dit que c'était pour cela que je ne me suis jamais sentie Française et que je suis peut-être perçue comme un peu bizarre parfois.

\section{$V:$ Le côté discipliné aussi ?}

PK : Non, car contrairement à ce que croit le reste du monde, le Suisse n'est pas discipliné, il veut montrer l'exemple. Il y a aussi cette idée qu'on doit faire sa part et assumer ce qu'on fait. En France, quand il neige et que les voitures se retrouvent en travers de la route, les gens invectivent le gouvernement, moi je me dis, mais pourquoi les gens ne mettent pas de pneus neige ? Je suis convaincue que nous avons tous une part de responsabilité dans la façon dont nos sociétés évoluent. C'est notre façon de consommer, d'élever nos enfants, de voter qui fait le monde tel qu'il est. Et ça, c'est une idée très suisse !

\section{$V:$ Et tes engagements dans ta vie reflètent aussi cette conviction?}

PK : Oui, mais c'est venu petit à petit, et par hasard. J'ai été amenée à faire des sortes d'ateliers d'écriture dans un centre d'hébergement de jour pour femmes et enfants à la rue. J'ai découvert ce monde des gens en marge et je m'y suis sentie bien. Plus tard, quand l'occasion s'est présentée d'aller habiter dans une colocation solidaire, avec des personnes qui viennent de la rue, j'ai tenté l'expérience, et j'ai adoré vivre avec ces personnes qui ont eu des vies folles et avec qui les liens sont très différents. En raison de ma dyslexie, et du fait que je n'ai pas fait d'études, je me sens rapidement en danger avec ceux qui maîtrisent parfaitement la langue, qui ont tout lu, tout retenu. J'ai toujours peur de dire un mot ou un nom pour un autre. C'est ainsi sans doute mon propre parcours qui m'a portée vers des gens, des milieux plus simples où, finalement, je me sens plus en confiance. Où il n'y a pas d'enjeu, où on a qu'à montrer qu'on s'aime, ce qui est déjà bien. D'aller vivre dans ce foyer, à huit nanas dans un seul appartement et avec une seule salle de bains, pour moi qui suis une célibataire endurcie, c'était un défi. Je me suis rendu compte que j'étais très adaptable, que je pouvais assez facilement réduire ma zone de confort. C'est une petite victoire. Après cette expérience, je me suis installée dans un nouvel appartement avec une petite chambre qui m'a permis d'héberger d'abord un Afghan, puis un Soudanais pendant assez longtemps. Et ça m’a entraîné encore ailleurs, notamment à m'engager dans l'association créée par le jeune Afghan. Je l'ai fait au départ par pure conviction (je suis pour l'accueil des réfugiés, donc à moi aussi de les accueillir) et cela m'a apporté de très grandes joies. J'aimerais convaincre 
d'autres personnes d'accueillir des réfugiés chez elles, cela contribuerait à faire retomber la tension. J'ai peur qu'en France l'extrême droite finisse par accéder au pouvoir et que ça se termine mal. Autopsie d'un père se termine par un embrasement. Je pense que le danger est réel et que ça ne suffit plus de s'indigner. Je suis un peu agacée par toutes ces pétitions sur Facebook. Cliquer pour sauver les migrants en mer, c'est bien, mais ensuite qui s'en occupe concrètement, qui est d'accord de leur faire une place? J'ai été récemment invitée à une conférence de presse à l'occasion de la sortie d'une étude réalisée par des associations parisiennes sur la prise en charge de l'hébergement des réfugiés. Leur propos consistait à dire que l'État se défausse sur les associations et sur les citoyens. J'ai prévenu les organisateurs que je voulais bien témoigner, mais que je dirai que selon moi, les citoyens aussi doivent faire leur part. L'État ne peut pas tout faire, et surtout pas la même chose. On se complète.

\section{$V:$ Alors que peut la littérature pour toi dans ce monde?}

$\mathrm{PK}$ : Vaste question. J'ai revu récemment une interview de Philip Roth à qui on pose cette question. Il répond que le rôle de l'écrivain, c'est de faire le mieux possible, que la littérature ne peut pas grand-chose, mais qu'elle est indispensable. J'aime bien cette réponse. La littérature, ça reste le seul lieu où on a le temps, la place, pour dire les choses dans toute leur complexité. C'est un espace de parole irremplaçable. Mes livres ne peuvent pas grandchose pour ce monde. Quand on vend à quelques milliers d'exemplaires, l'impact est dérisoire. Mais le simple fait d'essayer de comprendre les choses en profondeur par l'écriture me fait progresser moi dans ma vie et dans mes engagements. L'écriture permet d'aller plus loin dans la réflexion. C'est important que cela continue à exister. J'ai eu récemment une réunion avec les organisateurs du Salon du livre de Genève. On peut s'interroger sur l'avenir de ces manifestations. Il faut pourtant lutter contre la tentation d'aller vers des auteurs qui plaisent au public et qui amènent du public. Il faut continuer à faire entendre des voix peut-être moins consensuelles, mais qui ont leur importance. Le besoin d'exigence ne se perdra jamais complètement. Et puis, quand on ne pourra plus voyager aux quatre coins de la planète et qu'on n'aura plus le droit de cliquer aussi souvent qu'aujourd'hui, on sera peut-être obligé de revenir à la littérature, à la lecture. 
Ursula Bähler, Jean-Philippe Coen \& Pascale Kramer 
ENTRETIEN

Versants 67:I, fascicule français, 2020, pp. 95-III. 
Ursula Bähler, Jean-Philippe Coen \& Pascale Kramer

II4

Versants 67:I, fascicule français, 2020, pp. 95-III. 
ENTRETIEN

Versants 67:I, fascicule français, 2020, pp. 95-III. 
Ursula Bähler, Jean-Philippe Coen \& Pascale Kramer 
ENTRETIEN

Versants 67:I, fascicule français, 2020, pp. 95-III. 
Ursula Bähler, Jean-Philippe Coen \& Pascale Kramer 
ENTRETIEN

Versants 67:I, fascicule français, 2020, pp. 95-III. 
Ursula Bähler, Jean-Philippe Coen \& Pascale Kramer 
ENTRETIEN

Versants 67:I, fascicule français, 2020, pp. 95-III. 
Ursula Bähler, Jean-Philippe Coen \& Pascale Kramer 
ENTRETIEN

Versants 67:I, fascicule français, 2020, pp. 95-III. 
Ursula Bähler, Jean-Philippe Coen \& Pascale Kramer 
ENTRETIEN

Versants 67:I, fascicule français, 2020, pp. 95-III. 
Ursula Bähler, Jean-Philippe Coen \& Pascale Kramer 
ENTRETIEN

Versants 67:I, fascicule français, 2020, pp. 95-III. 
Ursula Bähler, Jean-Philippe Coen \& Pascale Kramer 
ENTRETIEN

Versants 67:I, fascicule français, 2020, pp. 95-III. 
Ursula Bähler, Jean-Philippe Coen \& Pascale Kramer 
ENTRETIEN

Versants 67:I, fascicule français, 2020, pp. 95-III. 
Ursula Bähler, Jean-Philippe Coen \& Pascale Kramer 
ENTRETIEN

Versants 67:I, fascicule français, 2020, pp. 95-III. 
Ursula Bähler, Jean-Philippe Coen \& Pascale Kramer 
ENTRETIEN

Versants 67:I, fascicule français, 2020, pp. 95-III. 
Ursula Bähler, Jean-Philippe Coen \& Pascale Kramer

I36

Versants 67:I, fascicule français, 2020, pp. 95-III. 
ENTRETIEN

Versants 67:I, fascicule français, 2020, pp. 95-III. 
Ursula Bähler, Jean-Philippe Coen \& Pascale Kramer

I38

Versants 67:I, fascicule français, 2020, pp. 95-III. 
ENTRETIEN

Versants 67:I, fascicule français, 2020, pp. 95-III. 
Ursula Bähler, Jean-Philippe Coen \& Pascale Kramer 
ENTRETIEN

Versants 67:I, fascicule français, 2020, pp. 95-III. 
Ursula Bähler, Jean-Philippe Coen \& Pascale Kramer 
ENTRETIEN

Versants 67:I, fascicule français, 2020, pp. 95-III. 
Ursula Bähler, Jean-Philippe Coen \& Pascale Kramer

I44

Versants 67:I, fascicule français, 2020, pp. 95-III. 
ENTRETIEN

Versants 67:I, fascicule français, 2020, pp. 95-III. 
Ursula Bähler, Jean-Philippe Coen \& Pascale Kramer

Versants 67:I, fascicule français, 2020, pp. 95-III. 
ENTRETIEN

Versants 67:I, fascicule français, 2020, pp. 95-III. 
Ursula Bähler, Jean-Philippe Coen \& Pascale Kramer

I48

Versants 67:I, fascicule français, 2020, pp. 95-III. 
ENTRETIEN

Versants 67:I, fascicule français, 2020, pp. 95-III. 
Ursula Bähler, Jean-Philippe Coen \& Pascale Kramer

Versants 67:I, fascicule français, 2020, pp. 95-III. 
ENTRETIEN

Versants 67:I, fascicule français, 2020, pp. 95-III. 
Ursula Bähler, Jean-Philippe Coen \& Pascale Kramer 
ENTRETIEN

Versants 67:I, fascicule français, 2020, pp. 95-III. 
Ursula Bähler, Jean-Philippe Coen \& Pascale Kramer

I54

Versants 67:I, fascicule français, 2020, pp. 95-III. 
ENTRETIEN

Versants 67:I, fascicule français, 2020, pp. 95-III. 
Ursula Bähler, Jean-Philippe Coen \& Pascale Kramer

I56

Versants 67:I, fascicule français, 2020, pp. 95-III. 
ENTRETIEN

Versants 67:I, fascicule français, 2020, pp. 95-III. 
Ursula Bähler, Jean-Philippe Coen \& Pascale Kramer

I58

Versants 67:I, fascicule français, 2020, pp. 95-III. 
ENTRETIEN

Versants 67:I, fascicule français, 2020, pp. 95-III. 
Ursula Bähler, Jean-Philippe Coen \& Pascale Kramer

I60

Versants 67:I, fascicule français, 2020, pp. 95-III. 
ENTRETIEN

Versants 67:I, fascicule français, 2020, pp. 95-III. 
Ursula Bähler, Jean-Philippe Coen \& Pascale Kramer 
ENTRETIEN

Versants 67:I, fascicule français, 2020, pp. 95-III. 
Ursula Bähler, Jean-Philippe Coen \& Pascale Kramer

I64

Versants 67:I, fascicule français, 2020, pp. 95-III. 
ENTRETIEN

Versants 67:I, fascicule français, 2020, pp. 95-III. 
Ursula Bähler, Jean-Philippe Coen \& Pascale Kramer

Versants 67:I, fascicule français, 2020, pp. 95-III. 
ENTRETIEN

Versants 67:I, fascicule français, 2020, pp. 95-III. 
Ursula Bähler, Jean-Philippe Coen \& Pascale Kramer

Versants 67:I, fascicule français, 2020, pp. 95-III. 
ENTRETIEN

Versants 67:I, fascicule français, 2020, pp. 95-III. 
Ursula Bähler, Jean-Philippe Coen \& Pascale Kramer 
ENTRETIEN

Versants 67:I, fascicule français, 2020, pp. 95-III. 
Ursula Bähler, Jean-Philippe Coen \& Pascale Kramer 
ENTRETIEN

Versants 67:I, fascicule français, 2020, pp. 95-III. 
Ursula Bähler, Jean-Philippe Coen \& Pascale Kramer

I74

Versants 67:I, fascicule français, 2020, pp. 95-III. 
ENTRETIEN

Versants 67:I, fascicule français, 2020, pp. 95-III. 
Ursula Bähler, Jean-Philippe Coen \& Pascale Kramer

Versants 67:I, fascicule français, 2020, pp. 95-III. 
ENTRETIEN

Versants 67:I, fascicule français, 2020, pp. 95-III. 
Ursula Bähler, Jean-Philippe Coen \& Pascale Kramer

I78

Versants 67:I, fascicule français, 2020, pp. 95-III. 
ENTRETIEN

Versants 67:I, fascicule français, 2020, pp. 95-III. 
Ursula Bähler, Jean-Philippe Coen \& Pascale Kramer

Versants 67:I, fascicule français, 2020, pp. 95-III. 
ENTRETIEN

Versants 67:I, fascicule français, 2020, pp. 95-III. 
Ursula Bähler, Jean-Philippe Coen \& Pascale Kramer 
ENTRETIEN

Versants 67:I, fascicule français, 2020, pp. 95-III. 
Ursula Bähler, Jean-Philippe Coen \& Pascale Kramer

I84

Versants 67:I, fascicule français, 2020, pp. 95-III. 
ENTRETIEN

Versants 67:I, fascicule français, 2020, pp. 95-III. 
Ursula Bähler, Jean-Philippe Coen \& Pascale Kramer

Versants 67:I, fascicule français, 2020, pp. 95-III. 
ENTRETIEN

Versants 67:I, fascicule français, 2020, pp. 95-III. 
Ursula Bähler, Jean-Philippe Coen \& Pascale Kramer

Versants 67:I, fascicule français, 2020, pp. 95-III. 
ENTRETIEN

Versants 67:I, fascicule français, 2020, pp. 95-III. 
Ursula Bähler, Jean-Philippe Coen \& Pascale Kramer

Versants 67:I, fascicule français, 2020, pp. 95-III. 
ENTRETIEN

Versants 67:I, fascicule français, 2020, pp. 95-III. 
Ursula Bähler, Jean-Philippe Coen \& Pascale Kramer 
ENTRETIEN

Versants 67:I, fascicule français, 2020, pp. 95-III. 
Ursula Bähler, Jean-Philippe Coen \& Pascale Kramer 
ENTRETIEN

Versants 67:I, fascicule français, 2020, pp. 95-III. 
Ursula Bähler, Jean-Philippe Coen \& Pascale Kramer

Versants 67:I, fascicule français, 2020, pp. 95-III. 
ENTRETIEN

Versants 67:I, fascicule français, 2020, pp. 95-III. 